\title{
Symptomatic Extraperitoneal Bladder Perforation Following Transurethral Bladder Surgery: Imaging with CT Urography
}

\author{
Lorenzo Mannelli, Joel A. Gross, Jonathan R. Medverd, Puneet Bhargava, Sarah Bastawrous
}

Department of Radiology, University of Washington (LM, JAG, JRM, PB, SB) and Department of Radiology, VA Puget Sound Medical Center (JRM, PB, SB), Seattle, WA, USA

\section{CASE}

An 80 year-old man presented to his primary care physician with painless gross hematuria. He reported having intermittent episodes of pink urine containing small clots for about three months. He was initially treated with a single course of ciprofloxacin for presumed urinary tract infection. His symptoms did not improve with antibiotic therapy and he was then referred to our institution for a computed tomography (CT) urogram. CT urogram showed a 4 x 3.6 centimeter $(\mathrm{cm})$ infiltrating solid mass along the left lateral posterior wall of the urinary bladder (Figure-1). Tumor also extended along the bladder dome. Prostate enlargement and bladder diverticula were also present. He was referred to the urology service and subsequently underwent transurethral resection of bladder tumor (TURBT) with fulguration. No intraoperative complications were noted and he returned to the recovery room in satisfactory condition on continuous bladder irrigation. Pathologic examination revealed a $5 \mathrm{~cm}$ transitional cell carcinoma with high grade growth pattern invading the deep muscular layer.

On postoperative day one, the patient reported abdominal pain and general discomfort. Physical exam revealed a grossly distended abdomen which was tender to palpation with positive guarding and decreased bowel sounds. He

Figure 1 - Axial CT urogram excretory phase images show a lobulated mass along the left wall of the urinary bladder (white arrow).

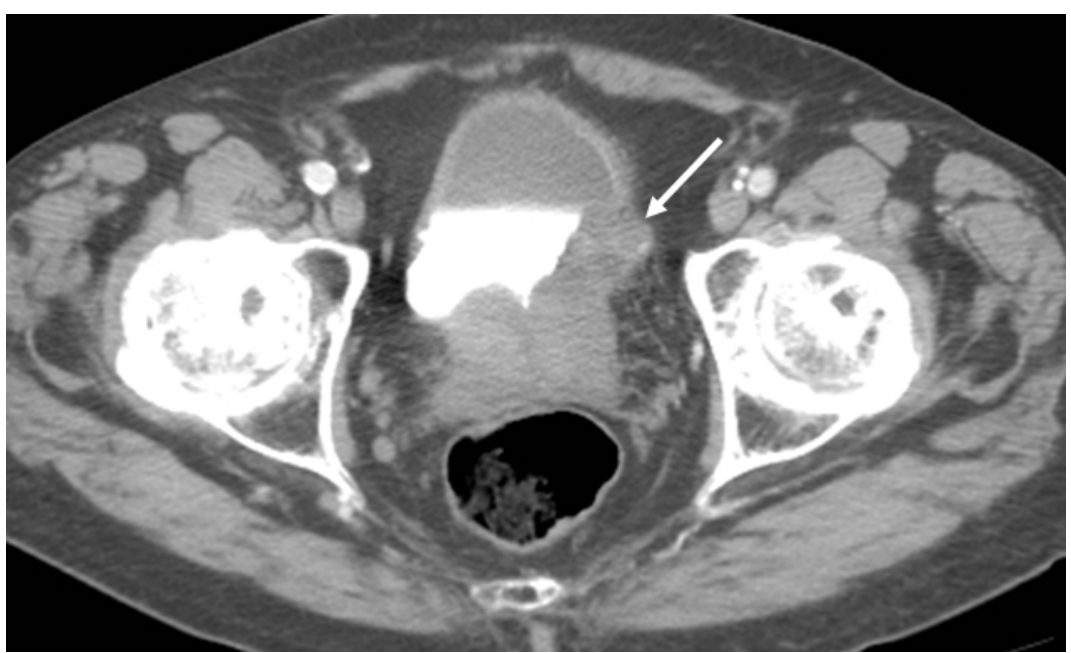


remained hemodynamically stable and bladder irrigation was pink in color without clots. At this time, bladder perforation was suspected and urgent CT urogram with multiplanar reformation (MPR) was performed. CT urogram showed irregular pooling of extravasated contrast into the left perivesical space via a disrupted left inferior lateral bladder wall, consistent with extraperitoneal bladder perforation (Figure-2). Bladder irrigation was discontinued and intravenous antibiotics along with catheterized bladder drainage were initiated. Our patient did well with conservative management and was discharged home on postoperative day four with oral antibiotics and an indwelling bladder catheter. CT urogram was performed two weeks later for assessment of bladder integrity prior to catheter removal and showed no evidence of leak or extravasation.

\section{DISCUSSION}

TURBT is the primary treatment option for bladder carcinoma, and is associated with few potential complications. Hemorrhage is the most frequent, and bladder perforation is the next most common complication. The actual incidence of bladder perforation is difficult to estimate as small perforations remain unnoticed, however one series reports an incidence of 1.3\%. Bladder perforations may be extraperitoneal or intraperitoneal and can be a consequence of inadvertent full thickness bladder wall resection during surgery. Extraperitoneal perforations are much more common and typically encountered with resection of posterior and lateral wall tumors, such as with our case (1). Extraperitoneal perforations are confined to the extraperitoneal space

Figure 2: A, B and C - CT urogram performed on postoperative day one following TURBT. (A) Axial pre contrast image shows high density material within the bladder, surrounding the catheter balloon compatible with intravesical clot. Gas is also present along the left lateral bladder. (B) After retrograde administration of contrast, axial CT image shows immediate pooling of contrast outside of the bladder compatible with extraperitoneal perforation (white arrow). (C) Coronal MPR image shows the irregular collection of contrast extravasation along the left inferior lateral margin of the bladder (white arrow).
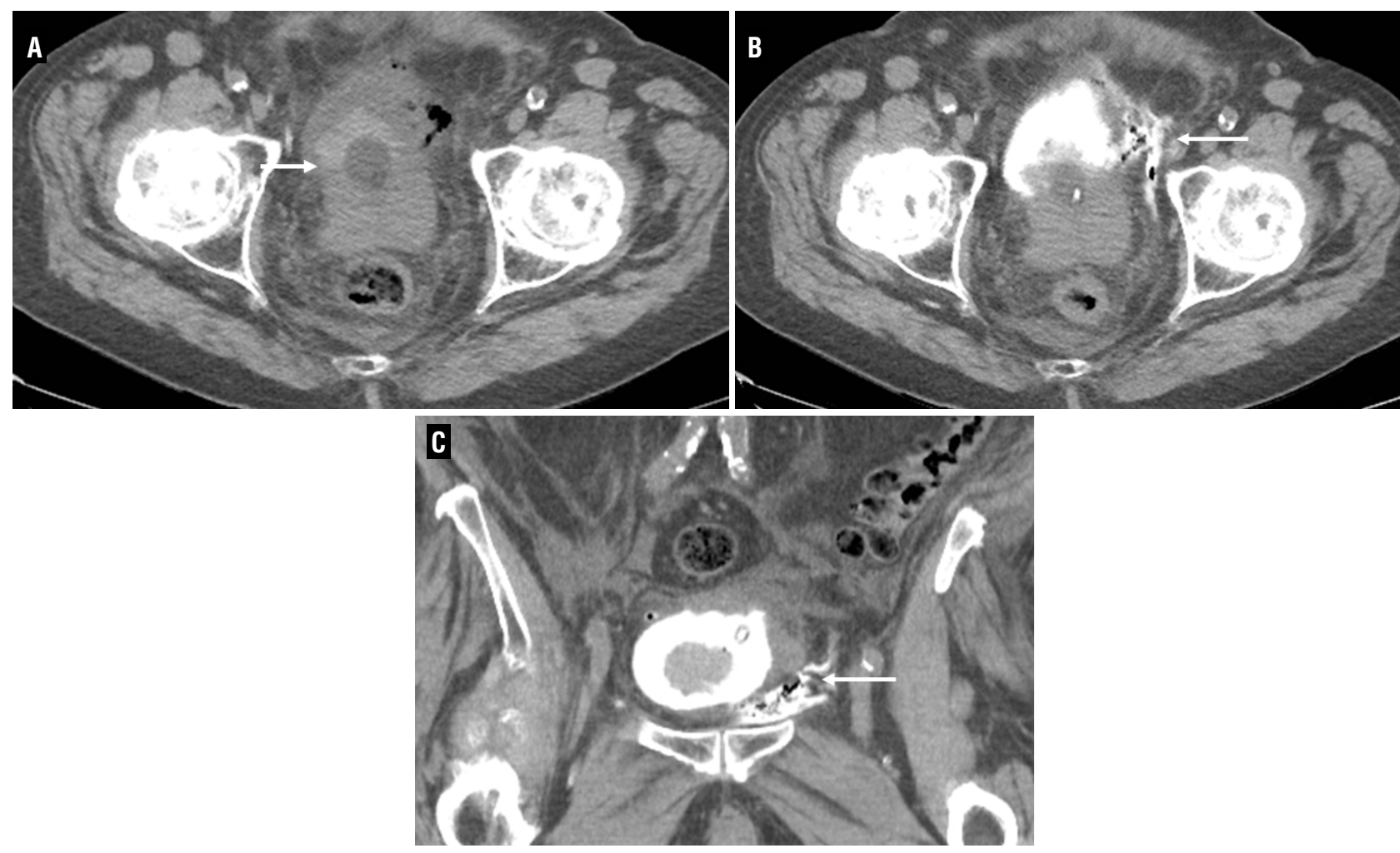
and imaging shows extravasation of contrast material close to the bladder, usually anteriorly, in a streaky and irregular pattern. Contrast leakage is confined to the extraperitoneal space, however complex cases may show extravasation extending to the thigh, scrotum or perineum and dissecting along fascial planes (2).

The diagnosis of iatrogenic bladder perforation during bladder surgery is generally established intraoperatively. In some cases however, it is not suspected until the perioperative period and confirmation can be obtained by performing CT urography. It is crucial to follow adequate CT urogram protocols that guarantee sufficient distention of the bladder as well as obtaining post-void CT images that will allow for the detection of small leaks (2). Prompt and accurate diagnosis of bladder perforation following transurethral bladder surgery is essential for appropriate management and a delay in diagnosis may increase patient morbidity and mortality.

Initial therapy for bladder perforation is adequate bladder drainage, with only a small percentage of patients requiring surgery $(1,3)$. In patients following TURBT, CT urography is a useful modality to evaluate complications such as perforation and to delineate relevant post surgical anatomy. This case demonstrates an initially unsuspected case of extraperitoneal bladder per- foration following TURBT, which was diagnosed by imaging. CT cystography is a reliable and fast method to determine the presence and location of suspected bladder perforation and to help guide appropriate therapy (4-6). For the detection of small dehiscences and non trasmural injuries post-voiding CT post-void phase after lavaging the bladder can be of used (7).

\section{REFERENCES}

1. Balbay MD, Cimentepe E, Unsal A, Bayrak 0, Koç A, Akbulut Z: The actual incidence of bladder perforation following transurethral bladder surgery. J Urol. 2005; 174: 2260-2, discussion 2262-3.

2. Vaccaro JP, Brody JM: CT cystography in the evaluation of major bladder trauma. Radiographics. 2000; 20: 1373-81.

3. Pansadoro A, Franco G, Laurenti C, Pansadoro V: Conservative treatment of intraperitoneal bladder perforation during transurethral resection of bladder tumor. Urology. 2002; 60: 682-4.

4. Ishak C, Kanth N: Bladder trauma: multidetector computed tomography cystography. Emerg Radiol. 2011; 18: 321-7.

5. Lang EK, Nguyen Q, Zhang K: Enhanced computed tomograms provide accurate assessment of local extension and stage of bladder carcinoma. Int Braz J Urol. 2012; 38: 129-31.

6. Zacharias C, Robinson JD, Linnau KF, Mannelli L: Blunt urinary bladder trauma. Curr Probl Diagn Radiol. 2012; 41: 140-1.

7. McLaughlin AP 3rd, Pfister RC: Double catheter technique for evaluation of urethral injury and differentiating urethral from bladder rupture. Radiology. 1974; 110: 716-9.

\section{ARTICLE INFO}

Int Braz J Urol. 2013; 39: 599-601

Submitted for publication:

August 09, 2012

Accepted after revision:

October 16, 2012

\footnotetext{
Correspondence address: Dr. Lorenzo Mannelli 325 Ninth Ave., Box 359728 Seattle, WA, 98104-2499, USA Fax: + 1206 744-8560 E-mail: mannellilorenzo@yahoo.it
} 